\title{
Nonlinear biharmonic equation with Hardy-Sobolev potential
}

\author{
Xibing $\mathrm{He}^{1}$, Yupei Zhang ${ }^{2}$ \\ ${ }^{1}$ Department of Mathematics and Computer Science, Nanchang Normal University, \\ Nanchang, Jiangxi, 330032, P. R. China \\ ${ }^{2}$ Nanchang Institute of Science and Technology, Nanchang, Jiangxi, 330108, P. R. China
}

Keywords: Biharmonic equation; Cerami condition; Fountain theorem; Sobolev-Hardy inequality

Abstract: This paper deals with the Dirichlet problem of a superlinear biharmonic equation with Hardy-Sobolev potential, where the nonlinearity is odd. By Fountain theorem with Cerami condition and Sobolev-Hardy inequality, we get the existence of nontrivial solutions for the problem.

\section{Introduction}

In this paper, we will consider the existence of multiple solutions of biharmonic equation with Dirichlet boundary Problem:

$$
\left\{\begin{array}{l}
\triangle^{2} u=\lambda \frac{u}{|x|^{s}}+f(x, u), \quad \text { in } \Omega, \\
u=\frac{\partial u}{\partial v}=0, \quad \text { on } \partial \Omega,
\end{array}\right.
$$

where $\Omega$ is a smooth bounded domain in $\mathbb{R}^{N}(N>4)$ and $0 \in \Omega, \Delta^{2}$ denotes the biharmonic operator, $\lambda>0$ is a parameter, $1 \leq s \leq 4, \frac{u}{|x|^{s}}$ is called Hardy-Sobolev potential.

In recent years, the problems with singularity have been a hot topic. But because of singularity, the problems are also difficult. In problem (1.1), if $f(x, u)=f(x)$ or $f(x, u)=|u|^{p-2} u, 2<p \leq \frac{2 N}{N-4}$ when $0 \leq s \leq 2$, Kang and Deng [5] studied the existence of the solutions of the problem (1.1) by using variational methods and Sobolev-Hardy inequalities. If $f(x, u)=|u|^{N-4} u$ in problem (1.1), by means of the concentrate compactness principle [6] and Mountain Pass theorem, Xiong and Shen [11] got the two existence results for the problem (1.1). Meanwhile, the authors proved, by Pohozaev identity for sigular solution, there is no nontrivial solution for equations with critical exponent and critical potential $[2,3,8]$. If $f(x, u)=f(x)+\frac{|u|^{2^{*}(\alpha)-2} u}{|x|^{\alpha}}, 2^{*}(\alpha)=\frac{2(N-\alpha)}{N-4}, 0 \leq \alpha<4$ and $s=4$ in problem (1.1), Hu and Song [4] got the existence of at least one solution under some certain condition by employing variational method and Sobolev inequality.

In this paper, our main interests are the problem (1.1) suggested by Kang and Deng [5], Liu and Li [7], Wang and Shen [9] and Xiong and Shen [11]. Our main methods follow that of Liu and Li [7] and Wang and Shen [9]. 
Throughout this paper, we denote the norms of $u$ in $H_{0}^{2}(\Omega)$ and $L^{p}(\Omega)$ by $\|u\|=\left(\int_{\Omega}|\Delta u|^{2} d x\right)^{1 / 2}$ and $|u|_{p}=\left(\int_{\Omega}|\Delta u|^{p} d x\right)^{1 / p}$, respectively. Here, $H_{0}^{2}(\Omega)$ is the Sobolev space $W_{0}^{2,2}(\Omega)$ with respect the norm $\|u\| . \rightarrow$ for weak convergence and $\rightarrow$ for strong convergence.

$u \in H_{0}^{2}(\Omega)$ is said to be a weak solution of the problem (1.1) if $u$ satisfies

$$
\int_{\Omega} \Delta u \Delta \varphi d x-\lambda \int_{\Omega} \frac{u}{|x|^{s}} \varphi d x-\int_{\Omega} f(x, u) \varphi d x=0, \forall \varphi \in H_{0}^{2}(\Omega) .
$$

It is well known that the notrivial solutions of the problem (1.1) are equivalent to the nonzero critical points of the energy functional

$$
I_{\lambda}(u)=\frac{1}{2} \int_{\Omega}|\Delta u|^{2} d x-\frac{\lambda}{2} \int_{\Omega} \frac{u^{2}}{|x|^{s}} d x-\int_{\Omega} F(x, u) d x,
$$

for $u \in H_{0}^{2}(\Omega)$, where $F(x, u)=\int_{0}^{u} f(x, s) d s$. So the Frechet dirivative $I^{\prime}(u)$ of the energy functional (1.2) at $u$ is

$$
\left\langle I^{\prime}(u), \varphi\right\rangle=\int_{\Omega} \Delta u \Delta \varphi d x-\lambda \int_{\Omega} \frac{u}{|x|^{\mid}} \varphi d x-\int_{\Omega} f(x, u) \varphi d x, \forall \varphi \in H_{0}^{2}(\Omega) .
$$

Let $\Phi(t, s)=\frac{1}{2} t^{\alpha} f(x, s) s-F(x, t s), \alpha \in(0,2)$ is a constant. Assume that function $f(x, s)$ satisfies

$\left(f_{1}\right) f(x, s) \in C(\bar{\Omega} \times \mathbb{R}, \mathbb{R}), f(x, 0)=0$ for any $x \in \Omega$;

$\left(f_{2}\right)$ there exists some constants $a, b>0$ such that $|f(x, s)| \leq a+b|s|^{q-1}$ for $(x, s) \in \Omega \times \mathbb{R}$, where $2<q<\frac{2 N}{N-4}$

$\left(f_{3}\right) \lim _{|s| \rightarrow \infty} \frac{f(x, s) s}{|s|^{2}}=\infty$ uniformly $x \in \Omega$;

$\left(f_{4}\right)$ there exist $\mu>0$ and $\delta>0$ such that $\Phi(t, s) \leq \mu \Phi(1, s)$, for any $x \in \Omega,|s| \geq \delta$ and $t \in(0,1]$;

$\left(f_{5}\right) f(x,-s)=-f(x, s), \forall(x, s) \in \Omega \times \mathbb{R}$

Assume $0 \leq s \leq 4$, then there exists a constant $C>0$ such that

$$
\left(\int_{\Omega} \frac{|u|^{q}}{|x|^{s}} d x\right)^{\frac{1}{q}} \leq C\left(\int_{\Omega}|\Delta u|^{2} d x\right)^{\frac{1}{2}}, \forall u \in H_{0}^{2}(\Omega) .
$$

We use $\lambda_{s}(\Omega)$ to denote the best Sobolev-Hardy constant, that is, the largest constant $C$ satisfying the above inequality for all $u \in H_{0}^{2}(\Omega)$, i.e.,

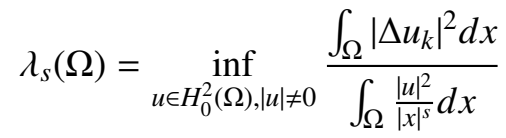

The main result of this paper is stated as follows.

Theorem 1.1 Let $\left(f_{1}\right)-\left(f_{5}\right)$ hold. If $\lambda<\lambda_{s}(\Omega)$, then problem (1.1) has infinitely many nontrivial solutions $\left\{u_{k}\right\}$ in $H_{0}^{2}(\Omega)$ which satisfies that

$$
\frac{1}{2} \int_{\Omega}\left|\Delta u_{k}\right|^{2} d x-\frac{\lambda}{2} \int_{\Omega} \frac{u_{k}^{2}}{|x|^{s}} d x-\int_{\Omega} F\left(x, u_{k}\right) d x \rightarrow+\infty \quad \text { as } \quad k \rightarrow+\infty .
$$




\section{Preliminaries}

Throughout this paper, we let $E$ be a real Banach space endowed with the norm $\|\cdot\|$ and $E^{*}$ the topological dual of $E$. For all $x \in E$ and $x^{*} \in E^{*}$, the value of $f$ at $x$ is denoted by $\langle x, f\rangle$ and is called the duality pairing. Then, we give the main definitions and lemmas in this section.

Definition 2.1 [10] Let $E$ be a real Banach space, a functional $I \in C^{1}(E, \mathbb{R})$ is said to satisfy the PalaisSmale condition ( (PS)-condition, for short) if there exists a constant $c \in \mathbb{R}$, any sequence $\left\{u_{n}\right\} \subset E$ satisfying

$$
I\left(u_{n}\right) \rightarrow c, I^{\prime}\left(u_{n}\right) \rightarrow 0, \text { as } \quad n \rightarrow \infty,
$$

possesses a convergent subsequence.

Definition 2.2 $[7,9]$ A functional $I \in C^{1}(E, \mathbb{R})$, where $E$ be a real Banach space, is said to satisfy the Cerami-condition $\left((C)\right.$-condition, for short) in $\left(c_{1}, c_{2}\right)\left(-\infty \leq c_{1}<c_{2} \leq+\infty\right)$ if for any $c \in\left(c_{1}, c_{2}\right)$,

(i) any bounded sequence $\left\{u_{n}\right\} \subset E$ satisfying (2.1) possesses a convergent subsequence, and

(ii) there exist $\delta, M, \epsilon>0$ such that $\left\|I^{\prime}\left(u_{n}\right)\right\|_{E^{*}}\left\|u_{n}\right\| \geq \epsilon$ for any $u \in I^{-1}[c-\delta, c+\delta]$ with $\left\|u_{n}\right\| \geq M$.

It is obvious that (C)-condition is slightly weaker than (PS)-condition while the most important implications of (PS)-condition are retained.

Let $E$ is a separable Banach space, and there exist $\left\{e_{n}\right\}_{n \in \mathbb{N}} \subset E$ and $\left\{\phi_{n}\right\}_{n \in \mathbb{N}} \subset E^{*}$ such that

(i) $\left\langle\phi_{n}, e_{m}\right\rangle=\delta_{n}^{m}$, where $\delta_{n}^{m}=1$ if $n=m, \delta_{n}^{m}=0$ if $n \neq m$, and

(ii) $\overline{\operatorname{span}}\left\{e_{n}, n \in \mathbb{N}\right\}=E, \overline{\operatorname{span}^{w^{*}}}\left\{\phi_{n}, n \in \mathbb{N}\right\}=E^{*}$.

Let $E_{j}=\operatorname{span}\left\{e_{j}\right\}$, then $E=\overline{\oplus_{j \geq 1} E_{j}}$. Set $Y_{k}=\overline{\oplus_{j=1}^{k} E_{j}}, Z_{k}=\overline{\oplus_{j \geq k} E_{j}}$. Thus, we have the following lemma:

Lemma 2.1 $[1,7,10]$ (Fountain theorem) Let $E$ be a real Banach space and $I \in C^{1}(E, \mathbb{R})$ with I even, that is, $I(-u)=I(u)$. Suppose $I(0)=0$ and $I$ satisfies $(C)$-condition, and for any $k \in \mathbb{N}$, there exits $\rho_{k}>r_{k}>0$ such that

(i) $b_{k}=\inf _{u \in Z_{k},\|u\|=r_{k}} I(u) \rightarrow+\infty$, as $k \rightarrow+\infty$, and

(ii) $a_{k}=\max _{u \in Y_{k},\|u\|=\rho_{k}} I(u) \leq 0$,

then I possesses an unbounded sequence of critical values.

Lemma 2.2 [12] Assume $1 \leq q \leq 2^{*}(s)=\frac{2(N-s)}{N-4}$, then 
(1) (Sobolev-Hardy inequality) for any $u \in H_{0}^{2}(\Omega)$, there exists a constant $C$ such that

$$
\left(\int_{\Omega} \frac{|u|^{q}}{|x|^{s}} d x\right)^{\frac{1}{q}} \leq C\left(\int_{\Omega}|\Delta u|^{2} d x\right)^{\frac{1}{2}}
$$

(2) the mapping $u \rightarrow \frac{u}{x^{s / q}}$ from $H_{0}^{2}(\Omega)$ into $L^{q}(\Omega)$ is compact provided $1 \leq q \leq 2^{*}(s)$.

Lemma 2.3 Assume $\left(f_{1}\right)-\left(f_{4}\right)$ hold, and suppose that there exists $\left\{u_{k}\right\} \subset H_{0}^{2}(\Omega)$ and $\left\{t_{k}\right\} \subset \mathbb{R}, t_{k}>0$ such that $\left\langle I^{\prime}\left(u_{k}\right), u_{k}\right\rangle \rightarrow 0$ and $t_{k} \rightarrow 0$ as $k \rightarrow \infty$. Let $w_{k}=t_{k} u_{k}$, then $I\left(w_{k}\right) \leq \mu I\left(u_{k}\right)+o(1)$ as $k \rightarrow \infty$.

Proof From the assumption of this lemma, it is easy to known that $t_{k}^{2}\left\langle I^{\prime}\left(u_{k}\right), u_{k}\right\rangle \rightarrow 0$ as $k \rightarrow \infty$, and consequently, we have

$$
\left\|w_{k}\right\|^{2}=\lambda \int_{\Omega} t_{k}^{2} \frac{u_{k}^{2}}{|x|^{s}} d x-\int_{\Omega} t_{k}^{2} f\left(x, u_{k}\right) u_{k} d x+o(1) .
$$

Hence, from $\left(f_{4}\right)$, we have

$$
I\left(w_{k}\right)=\frac{1}{2} \int_{\Omega} t_{k}^{2} f\left(x, u_{k}\right) u_{k} d x-\int_{\Omega} F\left(x, u_{k}\right) d x+o(1) \leq \mu \int_{\Omega} \Phi\left(x, u_{k}\right) d x+o(1)=\mu I\left(u_{k}\right)+o(1) .
$$

Lemma 2.4 Assume that $\left(f_{1}\right)-\left(f_{4}\right)$ hold, if $\lambda<\lambda_{s}(\Omega)$, then functional I satisfies $(C)$-condition.

Proof By Sobolev embedding theorem, we known that $H_{0}^{2}(\Omega) \rightarrow L^{q}(\Omega), 1 \leq q<2^{*}=\frac{2 N}{N-4}$ is compact. Meanwhile, from Lemma 2.2, it is easy to show that $I$ satisfies (C)-condition (i).

Next, we prove that $I$ satisfies (C)-condition (ii). Assume that there exists a constant $c \in \mathbb{R}$ and for any sequence $\left\{u_{k}\right\} \subset E$ such that

$$
I\left(u_{k}\right) \rightarrow c,\left\|u_{k}\right\| \rightarrow \infty,\left\|I^{\prime}\left(u_{k}\right)\right\|_{E^{*}}\left\|u_{k}\right\| \rightarrow 0, \text { as } \quad k \rightarrow \infty .
$$

Obviously, from the assumptions above, we obtain that $\left\langle I^{\prime}\left(u_{n}\right), u_{n}\right\rangle \rightarrow 0$. For any constant $K>0$, set

$$
t_{k}=\frac{\sqrt{2 K}}{\left\|u_{k}\right\|}, w_{k}=t_{k} u_{k}=\frac{\sqrt{2 K} u_{k}}{\left\|u_{k}\right\|}
$$

It is from (2.3) that $\left\{w_{k}\right\}$ is bounded in $H_{0}^{2}(\Omega)$, and hence there exists $w \in H_{0}^{2}(\Omega)$ such that $w_{k} \rightarrow w$ in $H_{0}^{2}(\Omega) ; w_{k} \rightarrow w$ in $L^{q}(\Omega), 1 \leq q<2^{*} ; w_{k} \rightarrow w$, a.e. $x \in \Omega$.

We will show the contradiction for the following two cases:

Case a) Suppose $w \equiv 0$, then from $w_{k} \rightarrow w$, a.e. $x \in \Omega$, we have

$$
\int_{\Omega} F\left(x, w_{k}\right) d x \rightarrow 0, k \rightarrow \infty .
$$

Thus, by Sobolev-Hardy inequality, we have

$$
I\left(w_{k}\right) \geq \frac{1}{2}\left\|w_{k}\right\|^{2}-\frac{\lambda}{2} \int_{\Omega} \frac{\left|w_{k}\right|^{2}}{|x|^{s}} d x-C \geq \frac{\sigma}{2}\left\|w_{k}\right\|^{2}-C .
$$

where $\sigma=\frac{\lambda_{s}(\Omega)-\lambda}{\lambda_{s}(\Omega)}$. 
By Lemma 2.3, we have

$$
\left\|w_{k}\right\|^{2} \leq \frac{2}{\sigma} I\left(w_{k}\right)+C \leq \frac{2 \mu}{\sigma} I\left(w_{k}\right)+C \leq C_{0} .
$$

Let $K=C_{0}$ in (2.3), then $\left\|w_{k}\right\|^{2}=2 C_{0}$, this is a contrary to (2.4).

Case b) Suppose $w \neq \equiv$, then by (2.2), we have

$$
\int_{\Omega}\left|\Delta u_{k}\right|^{2} d x-\lambda \int_{\Omega} \frac{\left|u_{k}\right|^{2}}{|x|^{s}} d x-\int_{\Omega} f\left(x, u_{k}\right) u_{k} d x=\left\langle I^{\prime}\left(u_{k}\right), u_{k}\right\rangle=o(1), \forall \varphi \in H_{0}^{2}(\Omega) .
$$

Hence,

$$
\left\|u_{k}\right\|^{2}=\int_{\Omega}\left|\Delta u_{k}\right|^{2} d x \geq \int_{\Omega}\left|\Delta u_{k}\right|^{2} d x-\lambda \int_{\Omega} \frac{\left|u_{k}\right|^{2}}{|x|^{s}} d x=\int_{\Omega} f\left(x, u_{k}\right) u_{k} d x+o(1) .
$$

Let $\Omega_{0}=\{x \in \Omega \mid w(x)=0\}$, then $\left|\Omega \backslash \Omega_{0}\right|>0$, and consequently from the inequality above, we have

$$
1 \geq \int_{\Omega_{0}} f\left(x, u_{k}\right) \frac{u_{k}}{2 K\left|u_{k}\right|^{2}}\left|w_{k}\right|^{2} d x+\int_{\Omega \backslash \Omega_{0}} f\left(x, u_{k}\right) \frac{u_{k}}{2 K\left|u_{k}\right|^{2}}\left|w_{k}\right|^{2} d x+o(1) .
$$

Meanwhile, for any $x \in \Omega \backslash \Omega_{0}$, we have $\left|u_{k}\right|(x) \rightarrow+\infty$. Hence, by $\left(f_{3}\right)$, we get

$$
f\left(x, u_{k}\right) \frac{u_{k}}{2 K\left|u_{k}\right|^{2}}\left|w_{k}\right|^{2} \rightarrow+\infty, k \rightarrow+\infty .
$$

From $\left|\Omega \backslash \Omega_{0}\right|>0$ and by Fatou Lemma, we have

$$
\int_{\Omega_{0}} f\left(x, u_{k}\right) \frac{u_{k}}{2 K\left|u_{k}\right|^{2}}\left|w_{k}\right|^{2} d x \rightarrow+\infty, k \rightarrow+\infty
$$

On the other hand, by $\left(f_{3}\right)$, we know that there exists $M>-\infty$ such that $\frac{f(x, s)}{s} \geq M, \forall(x, s) \in \Omega \times \mathbb{R}$. And we also note that $\int_{\Omega_{0}} \mid w_{k}(x)^{2} d x \rightarrow 0$ as $k \rightarrow+\infty$. Furthermore, we can find $\Lambda>-\infty$ such that

$$
\int_{\Omega_{0}} f\left(x, u_{k}\right) \frac{u_{k}}{2 K\left|u_{k}\right|^{2}}\left|w_{k}\right|^{2} d x \geq \frac{M}{2 K} \int_{\Omega_{0}}\left|w_{k}\right|^{2} d x>\Lambda>-\infty .
$$

Therefore, from (2.5)-(2.7), we deduce a contradiction.

Thus, we obtain that functional $I$ satisfies (C)-condition.

Lemma 2.5 If $\alpha \in\left[1,2^{*}(s)\right)$, Then $\beta_{k}:=\sup \left\{|u|_{\alpha}: u \in Z_{k},\|u\|=1\right\} \rightarrow 0 \quad(k \rightarrow+\infty)$.

Proof It is clear that $0<\beta_{k+1} \leq \beta_{k}$, so that $\beta_{k} \rightarrow \beta \geq 0$ as $k \rightarrow+\infty$. For every $k \geq 0$, there exists $u_{k} \in Z_{k}$ such that $\left\|u_{k}\right\|=1$ and $\left|u_{k}\right|_{\alpha}>\beta_{k} / 2$. By definition of $Z_{k}, u_{k} \rightarrow 0$ in $H_{0}^{2}(\Omega)$. The Sobolev imbedding theorem implies that $u_{k} \rightarrow 0$ in $L^{p}(\Omega)$. Thus we have proved that $\beta=0$.

\section{Proof of Theorem 1.1}

The main results of this paper are stated as follows.

Proof of Theorem 1.1 Firstly, by $\left(f_{5}\right)$, we obtain that $I(-u)=I(u)$ for any $u \in H_{0}^{2}(\Omega)$, and then it is from Lemma 2.4 that $I$ satisfies (C)-condition. 
Next, it is from $\left(f_{2}\right)$ that there exists $C>0$ such that $|F(x, s)| \leq C\left(1+|s|^{q}\right)$. Let $\beta_{k}:=\sup \left\{|u|_{q}: u \in\right.$ $\left.Z_{k},\|u\|=1\right\}, \quad k=1,2, \cdots$, then, by Lemma $2.5, \beta_{k} \rightarrow 0$ as $k \rightarrow+\infty$. Set $r_{k}=\left(\frac{4 C}{\sigma} \beta_{k}^{q}\right)^{\frac{1}{2-q}}$. If for $u \in Z_{k}$ such that $\|u\|=r_{k}$, then we have

$$
I(u) \geq \frac{\sigma}{2}\|u\|^{2}-C \beta_{k}^{q}\|u\|^{q}-C|\Omega|=\frac{\sigma}{2} r_{k}^{2}-C \beta_{k}^{q} r_{k}^{q}-C|\Omega|=\frac{\sigma}{4}\left(\frac{4 C}{\sigma}\right)^{\frac{2}{2-q}} \beta_{k}^{2 q /(2-q)}-C|\Omega|,
$$

Note that $\beta_{k} \rightarrow 0$ and $q>2$, from the above inequality, we have

$$
b_{k}=\inf _{u \in Z_{k},\|u\|=r_{k}} I(u) \rightarrow+\infty, \text { as } \quad k \rightarrow+\infty .
$$

Finally, we show that $a_{k}=\max _{u \in Y_{k},\|u\|=\rho_{k}} I(u) \leq 0$. In fact, we definite the norm $\|u\|_{\lambda, 2}^{2}=\int_{\Omega}\left(|\Delta u|^{2}-\right.$ $\lambda\left(\frac{|u|^{2}}{|x|^{s}}\right) d x$. Since on the finite dimensional space $\operatorname{dim} Y_{k}$ all norms are equivalent, we obtain that $\|u\|^{2} \Leftrightarrow$ $\|u\|_{\lambda, 2}^{2}$. Hence, there exits $B_{k}, C_{k}>0$ such that for any $u \in Y_{k}$,

$$
\begin{gathered}
\frac{1}{2} \int_{\Omega}\left(|\Delta u|^{2}-\lambda \frac{|u|^{2}}{|x|^{s}}\right) d x=\frac{1}{2}\|u\|_{\lambda, 2}^{2} \leq C_{k}|u|_{2}^{2}=C_{k} \int_{\Omega}|u|^{2} d x, \\
\|u\|^{2} \leq B_{k}|u|_{2}^{2} .
\end{gathered}
$$

By $\left(f_{2}\right)$, there exits $R_{k}>0$ such that $F(x, s) \geq 2 C_{k}|s|^{2}$ as $|s|>R_{k}$. On the other hand, let $M_{k}=$ $\max \left\{0, \inf _{x \in \Omega,|s| \leq R_{k}} F(x, s)\right\}$ if $|s| \leq R_{k}$, then for any $(x, s) \in \Omega \times \mathbb{R}$, we have

$$
F(x, s) \geq 2 C_{k}|s|^{2}-M_{k}
$$

By (3.1)-(3.3), for any $u \in Y_{k}$, we have

$$
I(u)=\frac{1}{2}\|u\|_{\lambda, 2}^{2}-\int_{\Omega} F(x, u) d x \leq-C_{k}|u|_{2}^{2}+M_{k}|\Omega| \leq-\frac{C_{k}}{B_{k}}\|u\|^{2}+M_{k}|\Omega| .
$$

It is easy to see that there exits sufficiently large $\rho_{k}>0$ such that $a_{k}=\max _{u \in Y_{k},\|u\|=\rho_{k}} I(u) \leq 0$.

Therefore, by Lemma 2.1, functional $I$ has an unbounded sequence of critical values.

\section{Acknowledgements}

In this paper, the research was supported by Science and Technology Research Project of Jiangxi Provincial Department of Education (No. GJJ151250), Startup Foundation for Doctors of Nanchang Normal University (No. 2015027), Nature Science Foundation of China (No. 11661057).

\section{References}

[1] Bartsch T. Infinity many solutions of symmetric Dirichlet problem[J]. Nonlinear Analysis: Theory, Mathematics and Applications, 1993, 20(10): 1205-1216. 
[2] Edmunds DE, Fortunato D. Jannelli E. Critical exponents, critical dimensions and te biharmonic operator[J]. Archive for Rational Mechanics and Analysis, 1990, 112: 269-289.

[3] Ghoussoub N, Yuan C. Multiple solutions for quasilinear PDEs involving the critical Sobolev and Hardy exponents[J]. Transanctions of the American Mathematical Society, 2000, 352: 5703-5743.

[4] Hu AL, Song AL. Existence of solution for a singular biharmonic equation involving critical exponent (Chinese)[J]. Journal of Huazhong Normal University (Natural Science Edition), 2011, 45(2): $175-179$.

[5] Kang DS, Deng YB. Sobolev-Hardy inequalities and some critical biharmonic problems (Chinese)[J]. Acta Mathematica Sinica, 2003, 23A(1): 106-114.

[6] Lions PL. The concentration-compactness principle in the calculas of varations, The limit case, part I and part II[J]. Revista Mathemática Iberoamericana 1985, 1: 145-201, 223-283.

[7] Liu SB, Li SJ. Infinitely many solutions for a superlinear elliptic equation (Chinese)[J]. Acta Mathematica Sinica, 2003, 46(4): 625-630.

[8] Pucci P, Serrin J. Critical exponents and critical dimentions for polyharmonic operatoers[J]. Archive for Rational Mechanics and Analysis, 1990, 112: 269-289.

[9] Wang YJ, Shen YT. Nonlinear biharmonic equations with Hardy potential and critical parameter[J]. Journal of Mathematical Analysis and Applications, 2009, 355: 649-660.

[10] Willem M. Minimax Theorem[M]. Birkhäuser: Boston, 1996.

[11] Xiong H, Shen YT. Nonlinear biharmonic equations with critical potential[J]. Acta Mathematica Sinica, English series, 2005, 21(6): 1285-1294.

[12] Yao YX, Wang RX, Shen YT. Nontrivial solution for a class of semlinear biharmonic equation involving involving critical exponents[J]. Acta Mathematica Sinica, 2007, 27B(3): 509-514. 